\title{
Diffusion of isotopes in the annual layers of ice sheets
}

\author{
J. F. NyE \\ H. H. Wills Physics Laboratory, University of Bristol, Tyndall Avenue, Bristol BS8 1TL, England
}

\begin{abstract}
The annual layering in the Greenland and Antarctic ice sheets revealed by the $\delta^{18} \mathrm{O}$ record becomes less distinct with depth because of self-diffusion. However, the calculated diffusion rates are too slow to explain the observations. It is suggested that the presence of veins of liquid water increases the effective diffusion constant by a factor of about 20.
\end{abstract}

\section{OUTLINE OF THE PROBLEM AND THE SUGGESTED SOLUTION}

The oxygen isotope ratios used to identify the annual layers of the Antarctic and Greenland ice sheets gradually lose their initial contrast by self-diffusion through the solid ice. However, recent work (Johnsen and others, 1997) on ice from the GRIP core, Greenland, concludes that the usual processes of solid-state diffusion, including grain-boundary diffusion, are too slow to produce the observed loss of contrast. The diffusion constant in the $-32^{\circ} \mathrm{C}$ cold Holocene ice needs to be about 10 times greater than the measured single-crystal diffusion constant at the same temperature (Ramseier, $1967 \mathrm{a}, \mathrm{b})$ to explain the data. This note suggests a new process, not previously recognised, that will enhance the rate.

It depends on the presence within the ice of veins of unfrozen water at the three-grain junctions (Nye and Frank, 1973; Mader, 1992a, b; Nye, 1992). The veins form a continuous network that renders the ice slightly permeable to water. They do not freeze when the temperature is lowered because of two physical effects: one is lowering of the freezing point by the curvature of the ice-water interfaces at the triple junctions (the ice is convex towards the water); the other is the lowering of the freezing point by soluble impurities in the vein water. As the temperature is reduced the veins tend to freeze, but this increases the curvature, and so lowers the freezing point still further; also, because the ice lattice rejects impurities, they remain in the liquid, which becomes more concentrated. The result is that a new equilibrium is found at the lower temperature, with the veins narrower but still open.

To see the effect of the veins on the diffusion of isotope ratios, consider two layers with isotope ratios $\delta=\delta_{0}+\Delta$ and $\delta=\delta_{0}-\Delta$ separated by a distance $\lambda / 2$, where $\lambda$ is the spacing of the annual layers. The standard diffusion process is driven by the gradient $4 \Delta / \lambda$. However, suppose we conceptually suspend this process and connect the layers by water veins. Isotope diffusion within the veins will be so fast compared with solid-state diffusion that $\delta$ within them will reach the mean value $\delta_{0}$ virtually instantaneously (within hours or days). Thus, the rate-controlling process is now lateral diffusion within the layers towards and away from the veins, and the gradients driving this process are $\pm \Delta / r_{1}$, where $r_{1}$ is half the spacing of the veins, which is approx- imately the grain-size $b$. If $r_{1}<\lambda / 4$, that is $b<\lambda / 2$, diffusion by this process could outpace the standard process. On dimensional grounds one would expect the rate for the lateral process to be about $(\lambda / 2 b)^{2}$ times the rate for the conventional vertical diffusion process. In other words, the veins might short-circuit the usual vertical diffusion and the rate of approach to uniformity would then be controlled by how close a point is to a vein, rather than by the layer spacing. That is the suggestion.

\section{MORE DETAILED MODEL}

To examine the geometry more carefully, with both processes taking place at once, consider a long vertical cylinder of ice of outer radius $r_{1}$ and inner radius $r_{0}$. The inner cylindrical hole represents a vein, and on its surface $\delta$ is maintained constant at $\delta_{0}$. The radius of the outer cylindrical boundary is taken as $r_{1}=b / 2$, where $b$ is the grain-size. Diffusion of $\delta$ takes place with diffusion constant $D$ in the vertical $z$ direction, and also radially with the boundary condition $\partial \delta / \partial r=0$ on $r=r_{1}$, according to the equation

$$
\frac{\partial \delta}{\partial t}=D \nabla^{2} \delta=D\left(\frac{\partial^{2}}{\partial r^{2}}+\frac{1}{r} \frac{\partial}{\partial r}+\frac{\partial^{2}}{\partial z^{2}}\right) \delta .
$$

Writing $\partial(r, z, t)=F(r) Z(z) T(t)$, and substituting, separates the variables to give

$$
T^{\prime}=-D k T, \quad Z^{\prime \prime}=-k_{z}^{2} Z,
$$

and

$$
F^{\prime \prime}(r)+\frac{1}{r} F^{\prime}(r)=-k_{r}^{2} F(r),
$$

where the $k$ 's are constants satisfying $k=k_{r}{ }^{2}+k_{z}{ }^{2}$. Thus, a solution can be written

$$
\delta-\delta_{0}=A\left(\sin k_{z} z\right) F(r) \mathrm{e}^{-k D t} \quad(A=\text { constant })
$$

with $F(r)$ obeying Equation (3) with boundary conditions $F\left(r_{0}\right)=0, F^{\prime}\left(r_{1}\right)=0$. With the substitutions $k_{r} r=R$, $k_{r} r_{0}=R_{0}, k_{r} r_{1}=R_{1}$, Equation (3) becomes the Bessel equation

$$
F^{\prime \prime}(R)+\frac{1}{R} F^{\prime}(R)+F(R)=0,
$$

with boundary conditions $F\left(R_{0}\right)=0, F^{\prime}\left(R_{1}\right)=0$.

The factor $\sin k_{z} z$ in Equation (4) represents the annual layering. Thus we regard $k_{z}$ as prescribed, but $k_{r}$ (the lowest 
frequency that fits the boundary conditions) remains to be found, although it must be of order $1 / r_{1}$. The question will be the relative contributions of $k_{z}$ and $k_{r}$ to the decay constant $k$.

The general solution of Equation (5) is

$$
F(R)=J_{0}(R)+B Y_{0}(R), \quad(B=\text { constant })
$$

the other arbitrary constant being absorbed into $A$.

The boundary conditions then imply

$$
J_{0}\left(R_{0}\right)+B Y_{0}\left(R_{0}\right)=0
$$

and

$$
J_{0}^{\prime}\left(R_{1}\right)+B Y_{0}^{\prime}\left(R_{1}\right)=0,
$$

which is equivalent to

$$
J_{1}\left(R_{1}\right)+B Y_{1}\left(R_{1}\right)=0 .
$$

$r_{0}$ and $r_{1}$ are given. At $-30^{\circ} \mathrm{C}$ the expected vein size is about $r_{0}=1 \mu \mathrm{m}$ (Mader, 1992b). With the grain-size in Holocene ice observed to be about 3-4 $\mathrm{mm}$ (Thorsteinsson and others, 1997), we take $r_{1}=3.5 / 2=1.8 \mathrm{~mm}$.

Although $r_{0}$ and $r_{1}$ are given, $R_{0}$ and $R_{1}$ are not, because $k_{r}$ is unknown. $R_{0}$ is given in terms of $R_{1}$ by $R_{0}=\left(r_{0} / r_{1}\right) R_{1}$. Then, eliminating $B$ between Equations (7) and (9) gives the following implicit equation for $R_{1}$ :

$$
\frac{J_{0}\left[\left(r_{0} / r_{1}\right) R_{1}\right]}{Y_{0}\left[\left(r_{0} / r_{1}\right) R_{1}\right]}-\frac{J_{1}\left(R_{1}\right)}{Y_{1}\left(R_{1}\right)}=0,
$$

which is solved by iteration to give $R_{1}=0.544$. It follows that $k_{r}=R_{1} / r_{1}=302 \mathrm{~m}^{-1}$.

This is to be compared with $k_{z}$. For ice 10000 years old the layer spacing is $\lambda=0.1 \mathrm{~m}$, which gives $k_{z}=2 \pi / \lambda=$ $63 \mathrm{~m}^{-1}$. Thus, $k_{r}$, from the lateral diffusion process, makes a larger contribution to the rate constant $k=k_{r}{ }^{2}+k_{z}{ }^{2}$ than does $k_{z}$, from conventional vertical diffusion. $k_{r}$ increases $k$ by a factor $f=\left(k_{r}{ }^{2}+k_{z}{ }^{2}\right) / k_{z}{ }^{2}=24$; that is, the diffusion constant will appear to be 24 times greater than normal. The result depends almost entirely on the relative values of $r_{1}$ and $\lambda$, the dependence on the vein size $r_{0}$ being logarith- mic only. For a grain-size of $6 \mathrm{~mm}$ instead of $3.5 \mathrm{~mm}$, as taken above, the factor would be 8.7 .

Because $R_{1}$ is always roughly 0.5 in the range of interest, a useful approximation is to take $k_{r} \approx 1 / b$, which gives the simple formula $f \approx 1+\left(\lambda^{2} / 4 \pi^{2} b^{2}\right)$.

Samples taken from the interiors of the individual ice grains ought to give the best contrast.

S. J. Johnsen (personal communication) remarks that the excess diffusion only acts in relatively clean glacier ice like the Holocene ice; samples from the dusty, last glacial, ice show no evidence of it. He suggests that the veins may be blocked by dust particles in the latter case. Of course, any blockage would have to act on the diffusion process along the veins, not just on the bulk motion of the water.

\section{ACKNOWLEDGEMENT}

I am grateful to S. J. Johnsen for kindly supplying me with essential data on this problem.

\section{REFERENGES}

Johnsen, S. J. and 14 others. 1997. The $\delta^{18} \mathrm{O}$ record along the Greenland Ice Core Project deep ice core and the problem of possible Eemian climatic instability. 7. Geophys. Res., 102 (C12), 26,397-26,410.

Mader, H. M. 1992a. Observations of the water-vein system in polycrystalline ice. f. Glaciol., 38 (130), 333-347.

Mader, H. M. 1992b. The thermal behaviour of the water-vein system in polycrystalline ice. f. Glaciol., $38(130), 359-374$.

Nye, J. F. 1992. Water veins and lenses in polycrystalline ice. In Maeno, N. and T. Hondoh, eds. Proceedings of the International Symposium on the Physics and Chemistry of Ice, Sapporo, Japan. Sapporo, Hokkaido University Press, 200-205.

Nye, J. F. and F. C. Frank. 1973. Hydrology of the intergranular veins in a temperate glacier. International Association of Scientific Hydrology Publication 95 Symposium at Cambridge 1969 - Hydrology of Glaciers), 157-161.

Ramseier, R. O. 1967a. Self-diffusion in ice monocrystals. CRREL Res. Rep. 232.

Ramseier, R. O. 1967b. Self-diffusion of tritium in natural and synthetic ice monocrystals. 7. Appl. Phys., 38(6), 2553-2556.

Thorsteinsson, Th., J. Kipfstuhl and H. Miller. 1997. Textures and fabrics in the GRIP ice core. J. Geophys. Res., 102 (C12), 26,583-26,599.

MS received 24 October 1997 and accepted in revised form 5 January $1998^{*}$

* Publication of this paper was delayed due to its loss by the postal services. 